\title{
Prevalence and Predictors of Trichomonas Infection in Newly Incarcerated Women
}

\author{
Ank E. Nijhawan, MD, MPH ${ }^{\star}$, Kimberle C. Chapin, MD, D(ABMM), FCAP ${ }^{\dagger}$, Rachel Salloway, \\ BS $^{\ddagger}$, Sarah Andrea, BS $^{\dagger}$, Jessi Champion, MSPH $^{\S}$, Mary Roberts, MS ${ }^{\ddagger}$, and Jennifer G. \\ Clarke, MD, MPH ${ }^{\ddagger}$ \\ "Division of Infectious Diseases, University of Texas Southwestern Medical Center, Dallas, TX \\ tDepartment of Pathology, Rhode Island Hospital, Providence, RI \\ ¥Center for Primary Care and Prevention, Memorial Hospital of Rhode Island, Pawtucket, RI \\ $\S$ Department of Community Health, Brown University, Providence, RI
}

\begin{abstract}
Background-Trichomonas vaginalis is the most prevalent curable sexually transmitted infection in the United States and may lead to pre-term delivery, infertility, and increased HIV transmission. Incarcerated women may be at especially high risk for infection, although few studies have examined routine screening for Trichomonas infection in this population.
\end{abstract}

Methods-Women older than 18 years entering the Rhode Island Department of Corrections between September 2009 and May 2011 were recruited to participate. All women submitted a selfcollected vaginal swab for APTIMA transcription-mediated amplification testing. Each participant completed a survey addressing demographics, symptoms, sexual behavior, and substance use by audio computer-assisted self-interview. Data analysis was completed using multivariate logistic regression in SAS.

Results-Data for 387 women were analyzed. The mean age was 30 years, $60 \%$ were white, $18 \%$ were Hispanic, $10 \%$ were black, and $12 \%$ had other race/ethnicity. Forty-four percent reported vaginal symptoms, and 77\% reported illicit drug and/or heavy alcohol use in the 30 days before incarceration. The prevalence of Trichomonas was 14\% by APTIMA. The strongest predictors of infection included black race (odds ratio [OR], 5.1; 95\% confidence interval [CI], $1.9-13.4 ; P<0.01)$, more than 1 year since last Papanicolaou test (OR, 2.5; 95\% CI, $1.3-4.8 ; P<$ $0.01)$ and presence of vaginal symptoms (OR, $2.3 ; 95 \% \mathrm{CI}, 1.2-4.7 ; P=0.02)$.

Conclusions-Trichomonas infection is common in incarcerated women, especially among black women, women with vaginal symptoms, and those not receiving routine gynecologic care. Screening for Trichomonas infection in high-risk populations, particularly if using highly sensitive methods such as transcription-mediated amplification, may lead to increased detection and treatment.

Trichomonas infection is common in the United States, with an estimated 7.4 million infections annually. ${ }^{1}$ Historically, it has been overlooked relative to other sexually transmitted infections (STIs) because of suboptimal diagnostic methods and underrecognition of the consequences of infection. However, novel testing methods such as nucleic acid amplification are more sensitive than routinely available tests used for diagnosis

Copyright (C 2012 American Sexually Transmitted Diseases Association. All rights reserved.

Correspondence: Ank E. Nijhawan, MD, MPH, Division of Infectious Diseases, University of Texas Southwestern Medical Center, 5323 Harry Hines Blvd, Dallas, TX 75390-9113. Ank.Nijhawan@UTSouthwestern.edu. 
including saline wet mount and Trichomonas culture. ${ }^{2}$ Furthermore, Trichomonas infection has been associated with infertility, cervical dysplasia, and preterm delivery. ${ }^{3}$

Trichomoniasis may also cause increased HIV acquisition and transmission, ${ }^{4,5}$ emphasizing the public health implications for detection and treatment of this STI.

Among reproductive-aged women in the general US population, regardless of symptoms and using nucleic acid amplification testing, the prevalence of Trichomonas infection is $3.1 \%$ to $8.7 \% .{ }^{6,7}$ Certain subgroups have been reported to have much higher rates of disease, including black women who use drugs (38\%), ${ }^{8}$ HIV-infected women $(9 \%-30 \%),{ }^{9}$ and women recruited from the Internet $(10 \%) .{ }^{10}$ In a large multicenter cohort study, a history of incarceration was found to be independently associated with trichomoniasis (odds ratio [OR], 1.37; $P=0.04$ ), after adjusting for multiple-risk factors for STIs. ${ }^{11}$ Another study found that age, black race, testing in the emergency department, sexually transmitted disease clinic, or jail were significant predictors of trichomoniasis on multivariate analysis. ${ }^{7}$ Although routine screening in incarcerated women in San Francisco detected 32\% positive infections, ${ }^{12}$ no study has been performed to date in incarcerated women with both routine testing and collection of symptom and sociodemographic data.

The current study seeks to determine the prevalence and predictors of Trichomonas infection among newly incarcerated women using APTIMA analyte-specific reagents (Gen-Probe, San Diego, CA) specific for transcription-mediated amplification (TMA) of Trichomonas vaginalis. Defining predictors of Trichomonas infection in this population not only will improve our understanding of the epidemiology of this STI but also may also help guide prevention, screening, and treatment efforts in the future.

\section{MATERIALS AND METHODS}

\section{Recruitment}

The Rhode Island Department of Corrections (RIDOC) is a combined jail and prison facility in Cranston, RI, with an average number of commitments for women of approximately 2000 per year. Newly incarcerated female inmates at the RIDOC were recruited to 2 different studies from September 2009 to May 2011. The first study focused on Trichomonas infection alone, and all recently incarcerated female inmates older than 18 years were eligible. A second study, entitled Project Contraceptive Awareness and Reproductive Education (CARE), was aimed at incarcerated women at risk for unplanned pregnancy aged 18 to 35 years and also incorporated Trichomonas testing. For either study, participants had to be able to communicate in English, had to be able to provide consent for the study, and could not be housed in segregation. Of note, segregation is where inmates who have been violent or disruptive are located, usually for short periods, and accounts for about $2 \%$ to 5\% of the population. Traffic sheets, which record the names of women committed or released from the facility for the preceding day or weekend, were reviewed on weekdays by a research assistant, who then approached potential participants on the prison wing where they were housed to determine if they were interested in participating in either study.

For both studies, after obtaining informed consent, the research assistant instructed the participant on how to collect a self-obtained vaginal swab. The participants were then directed to the bathroom to obtain 2 self-collected vaginal swab samples. After collection, the research assistant placed one swab in Trichomonas culture media and the other swab in a transport tube (APTIMA; Gen-Probe). After swab collection, the participants were asked to complete a questionnaire using audio computer-assisted self-interview (ACASI) regarding demographic background (race, education, marital status, living situation), incarceration history, health care use, history of STIs, obstetric/gynecologic history, current symptoms, sexual history, alcohol and drug use history, and acceptability of self-collected swabs. For 
Project CARE participants, Gonorrhea and Chlamydia testing, in addition to Trichomonas testing, was conducted from the transport tube, and additional interview information regarding contraception and pregnancy attitudes was gathered. Project CARE participants had multiple visits, whereas those in the Trichomonas-only study had only 1 visit. For the purposes of the current analysis, only the initial visit for all participants, with corresponding Trichomonas results as determined by APTIMA and related ACASI responses, were included.

Both studies were approved by the institutional review board of the Memorial Hospital of Rhode Island and by the Medical Research Advisory Group at the RIDOC. In addition, a certificate of confidentiality was obtained from the federal government to ensure the privacy of participants.

\section{Laboratory Testing for Trichomonas}

T. vaginalis cultures for all samples were performed on-site at the RIDOC women's division using the InPouch TV test kit (BioMed Diagnostics, White City, OR). The samples were stored at $37^{\circ} \mathrm{C}$ and were read at 0,2 , and 5 days of inoculation by trained personnel. Swabs placed in APTIMA transport tubes were used for TMA. The samples were batched and delivered to the Miriam Hospital microbiology laboratory weekly. The APTIMA $T$. vaginalis (ATV) assay was performed by using the APTIMA general-purpose reagent and ATV analyte-specific reagent oligonucleotides (Gen-Probe), which were reconstituted as described previously. ${ }^{2}$ Before March 1, 2010, specimens were tested on a semiautomated direct tube sampling platform (as described in the APTIMA Combo 2 package insert; GenProbe Inc); after that date, the specimens were tested on the fully automated Tigris direct tube sampling platform. Probes, reagents, and interpretations are the same as previously described ${ }^{13}$ and are the same as those used in the recently Food and Drug Administration (FDA)-cleared ATV assay.

\section{Data Analysis}

Analyses included (1) summarizing baseline characteristics, (2) summarizing the primary outcome (TMA results), and (3) determining crude and adjusted relationships between baseline characteristics and the primary outcome. Multivariate logistic regression was used to determine patient characteristics that predict Trichomonas infection. The following independent variables were included in the analysis: race/ethnicity (white/non-Hispanic, black/non-Hispanic, Hispanic, other [Pacific Islander, Native American, multiracial]), age, education (binary, completed high school/general equivalency diploma [GED] vs. not), marital status (binary, living with partner/married vs. other [single, separated, divorced, widowed]), homelessness, health status (ordinal variable ranging from poor to excellent), health insurance, prior STIs, current symptoms (vaginal discharge, unusual or foul odor from the vagina, bleeding or pain after intercourse, dysuria, vaginal itching), ever pregnant, ever miscarried, last Papanicolaou (Pap) test more than 1 year prior, if participant identified a main and/or casual partner, condom use (ordinal) with main or casual partners (simplified to always and almost always vs. other), number of lifetime partners ( $<10$ or $\geq 10$ because this was a nonlinear variable), ever exchanged sex for drugs or money, and drug (heroin, other opiates, cocaine, benzodiazepines, marijuana) and alcohol use (using alcohol to intoxication or any drug use in the 30 days before incarceration was considered as positive for alcohol and drug use, respectively). Not all participants answered each question in the questionnaire, but because the number of missing data was small, the denominator used for data analysis was limited to those answering the question.

Several variables were included a priori in the multivariate model: age, race, and any reported symptoms. A multivariate model was then built incorporating all factors that were 
significant in univariate analyses, with a $P$ value of less than 0.05 , in addition to the a priori variables. All analyses were completed using SAS software, version 9.2 (SAS Institute Inc, Cary, NC).

\section{RESULTS}

Data from 423 women and 378 samples were included in this evaluation. Figure 1 displays the study inclusion algorithm. Baseline characteristics of participants are presented in Table 1. The mean age was 30 years, and most $(60 \%)$ were white. The mean level of education was 11th grade, and approximately half had some type of health insurance. Sixty one percent reported being diagnosed as having an STI in the past, including 2\% who reported being HIV infected. At the time of the questionnaire, $44 \%$ reported some type of vaginal or urinary symptom and 33\% reported sex with both women and men. There were high levels of drug use in this study: 77\% reporting any drug use (including alcohol) and 55\% reporting polysubstance use in the 30 days before incarceration.

The prevalence of Trichomonas infection by APTIMA TMA testing was 14\% (53/378). All samples that tested positive by the initial TMA were also positive by a TMA testing for an alternate TV sequence (100\%). Incidentally, all samples with positive culture results were positive by APTIMA TMA.

The results of the univariate and multivariate analyses for predictors of Trichomonas infection as detected by TMA are presented in Table 2. Significant predictors in univariate analyses were older age, black race, last Pap test more than 1 year prior, vaginal symptoms, having 10 or more lifetime sexual partners, having had sex in exchange for drugs or money, and recent cocaine use. Predictors that were significant in the multivariate adjusted model were black race (OR, 5.1; $95 \%$ confidence interval $[\mathrm{CI}], 1.9-13.4 ; P<0.01)$, more than 1 year since last Pap test (OR, 2.5; 95\% CI, 1.3-4.8; $P<0.01)$, and presence of vaginal symptoms (OR, 2.3; 95\% CI, 1.2-4.7; $P=0.02)$.

\section{CONCLUSIONS}

In this study, we found that $T$. vaginalis is highly prevalent in incarcerated women at a rate of $14 \%$ using the APTIMA assay. These rates are significantly higher than the rate of infection in the general US female population $(3.1 \%-8.1 \% \text { by TMA })^{6,7}$ and are between the rates reported in female prison inmates $(8.5 \%)^{14}$ and the high rates reported in California jails $(32 \%) .{ }^{12}$ Rhode Island is a relatively low prevalence state for STIs, and our findings emphasize the correctional setting as a high-yield location to identify and treat STIs such as $T$. vaginalis. In addition, by using a newly FDA-cleared sensitive testing method (ATV), we are better able to define the epidemiology of $T$. vaginalis.

The association with race was particularly strong, and in fact, after controlling for multiple other factors, black women had 5.1 times the odds of testing positive for T. vaginalis than did white women, despite comprising only $10 \%$ of the study population. Among blacks, the prevalence of Trichomonas infection was 25\% compared with 13\% among whites and 15\% among Hispanics. Prior studies indicate that $T$. vaginalis is more prevalent in blacks, ${ }^{7,15}$ and Miller et al. ${ }^{8}$ suggested that $T$. vaginalis may be endemic in this racial group. These results mirror the rates of other STIs in the United States, such as Neisseria gonorrhea, Chlamydia trachomatis, syphilis, and HIV, which affect a disproportionate number of non-Hispanic blacks compared with those of other ethnicities or races. ${ }^{16}$ The STI epidemic in the black community is complex and involves overlapping issues including socioeconomic factors, access to health care, substance use, ratio of men to women, and concurrency of partners. ${ }^{17}$ Incarceration is especially disruptive because 1 in 9 black men between the ages of 20 and 
34 years are incarcerated at any given time, ${ }^{18}$ which affects sexual networks and may connect individuals who were previously at low risk for STIs with high-risk partners. ${ }^{17}$

The control of T. vaginalis has implications beyond addressing a health disparity for an isolated STI. The high prevalence of Trichomonas among black women and the association between Trichomonas and HIV infection may play a significant role in the HIV epidemic. ${ }^{15}$ The presence of $T$. vaginalis may increase the risk of HIV acquisition by nearly 2 -fold. ${ }^{5} \mathrm{In}$ addition, Kissinger et al. ${ }^{19}$ have shown that treatment of Trichomonas reduces vaginal shedding of HIV in women who have both HIV and Trichomonas. Black women already account for $66 \%$ of HIV infections among adolescent and adult women in the United States, ${ }^{20}$ and their high rates of $T$. vaginalis increase their risk of acquiring or transmitting HIV. Chesson and colleagues ${ }^{21}$ estimated that each year, 746 new HIV cases in the United States can be attributed to Trichomonas infection, with an estimated lifetime treatment cost of Trichomonas-attributable HIV infection of \$167 million. Therefore, detection and treatment of $T$. vaginalis may have far-reaching effects on HIV prevention, particularly in minority populations.

Although symptoms were a strong predictor of outcome and this may help direct future screening efforts, many without any reported symptoms tested positive for $T$. vaginalis. If only those study participants with symptoms had been tested, at best $43 \%$ (162) of the total 378 individuals would have been tested, missing $24(45 \%)$ of positives. There is limited literature on the cost-effectiveness of $T$. vaginalis screening, although one suggested costsaving approach has been to perform culture alone or TMA only when wet mount is negative. ${ }^{22}$ With the FDA approval of the ATV assay, a testing algorithm that incorporates this more sensitive testing technique that can be used in conjunction with testing for the STIs of $C$. trachomatis and $N$. gonorrhea could be considered.

Many participants had not had Pap screening in more than a year and others in more than 3 years. The American College of Obstetrics and Gynecology's most recent recommendation is that women aged 21 to 30 years need cervical screening every 2 years and women older than 30 years need screening every 3 years if results from 3 consecutive Pap tests have been negative. ${ }^{23}$ However, incarcerated women are known to have high rates of cervical dysplasia, ${ }^{24}$ and prior data from the RIDOC showed that the strongest predictor of having a recent Pap test performed was being incarcerated. ${ }^{25}$ Therefore, it is likely that a large proportion of these high-risk women are not up to date on cervical screening and that incarceration is an important opportunity to provide preventive health care, including STI screening. Although a routine Pap test is not considered a sensitive test for T. vaginalis (approximately $60 \%$ sensitivity), ${ }^{26}$ organisms are often identified on this test, and it is recommended that women be treated when Trichomonas organisms are noted. Recent data showed that TMA testing using an endocervical swab in PreservCyt (solution used for Pap test collection) is highly sensitive for T. vaginalis. ${ }^{27}$

Our study has several limitations. The study was conducted at a single site, and therefore, the results may not be generalizable to other incarcerated women. However, the RIDOC does serve as the only jail and prison for the entire state of Rhode Island, so our data do reflect the epidemiology of Trichomonas in incarcerated women in this state. We did have a substantial number of women who refused to participate (Fig. 1), and although we do not know specifically how these women differed from those studied, we do know that our study population closely resembles the general female RIDOC population in terms of race $(60 \%$ vs. $62 \%$ white and $10 \%$ vs. $11 \%$ black, respectively), mean age (30 vs. 32 years), and education (37\% vs. $43 \%$ have less than a high school education). All questionnaire data are based on self-report, including sexual and substance use history, although the use of ACASI has been shown to decrease social desirability bias. ${ }^{28}$ Although women were given direct 
instructions on how to collect their own vaginal swabs, 8 samples $(2 \%)$ were considered "quantity not sufficient," suggesting that an inadequate sample was collected. Because this was a single-visit study, samples were not recollected in those noted to be insufficient. In addition, ATV is not currently FDA cleared for self-collection, but self-collected vaginal swabs have been demonstrated to be an adequate choice for screening for Chlamydia and Gonorrhea ${ }^{29}$ as well as Tri-chomonas. ${ }^{10} \mathrm{We}$ did not control for personal hygiene practices such as vaginal douching or test for bacterial vaginosis, which have both been associated with Trichomonas infection. ${ }^{6,30}$ Lastly, sexual partners of study participants were not routinely tested or treated as part of the study, although in practice, many of the participants' partners did receive treatment.

Until recently, the epidemiology of $T$. vaginalis has been poorly understood because of a lack of accessible, sensitive testing methods. This study confirms prior results and also extends our knowledge of the epidemiology of Trichomonas infection. T. vaginalis was more common in older women, black women, women who had not had a Pap test in over a year, women with vaginal symptoms, women with 10 or more lifetime sexual partners, women who had had sex in exchange for drugs or money, and recent cocaine users. After adjusting for all variables, only black race, Pap test greater than a year prior, and the report of any symptoms remained significant predictors of Trichomonas infection.

Given the extremely high rates of incarceration in the United States, the barriers to affordable and accessible quality health care for underserved populations and the ensuing socioeconomic and health disparities, it is imperative that incarcerated individuals receive health services during and after their incarceration. Screening and treatment of Trichomonas infection in this setting, particularly with the known association between trichomoniasis and HIV, are an important step in improving the health outcomes of disadvantaged individuals in jail and prison, as well as improving the health of the communities they return to upon release. Future directions include implementing universal STI screening programs in correctional settings and drug treatment programs, improving partner notification and treatment, and facilitating linkage to medical care after release.

\section{Acknowledgments}

Supported by American Sexually Transmitted Diseases Association Developmental Award (A.E.N.), National Institute of Child Health and Human Development 5R01HD054890-04 (J.G.C.). Gen-Probe donated reagents for transcription-mediated amplification testing. K.C.C. has received research funding from Gen-Probe.

\section{References}

1. Centers for Disease Control and Prevention. [Accessed March, 12, 2012.] STD fact sheetTrichomonas. Available at: http://www.cdc.gov/std/trichomonas/stdfact-trichomoniasis.htm

2. Nye MB, Schwebke JR, Body BA. Comparison of APTIMA Trichomonas vaginalis transcriptionmediated amplification to wet mount microscopy, culture, and polymerase chain reaction for diagnosis of trichomoniasis in men and women. Am J Obstet Gynecol. 2009; 200(2):188.e181188.e187. [PubMed: 19185101]

3. Cotch MF, Pastorek JG 2nd, Nugent RP, et al. Trichomonas vaginalis associated with low birth weight and preterm delivery. The Vaginal Infections and Prematurity Study Group. Sex Transm Dis. 1997; 24(6):353-360. [PubMed: 9243743]

4. McClelland RS, Sangare L, Hassan WM, et al. Infection with Trichomonas vaginalis increases the risk of HIV-1 acquisition. J Infect Dis. 2007; 195(5):698-702. [PubMed: 17262712]

5. Laga M, Manoka A, Kivuvu M, et al. Non-ulcerative sexually transmitted diseases as risk factors for HIV-1 transmission in women: Results from a cohort study. AIDS. 1993; 7(1):95-102. [PubMed: 8442924] 
6. Sutton M, Sternberg M, Koumans EH, et al. The prevalence of Trichomonas vaginalis infection among reproductive-age women in the United States, 2001-2004. Clin Infect Dis. 2007; 45(10): 1319-1326. [PubMed: 17968828]

7. Ginocchio CC, Chapin K, Smith JS, et al. Prevalence of Trichomonas vaginalis and coinfection with Chlamydia trachomatis and Neisseria gonorrhoeae in the United States as determined by the APTIMA(R) Trichomonas vaginalis nucleic acid amplification assay. J Clin Microbiol. 2012; 50(8):2601-2608. [PubMed: 22622447]

8. Miller M, Liao Y, Gomez AM, et al. Factors associated with the prevalence and incidence of Trichomonas vaginalis infection among African American women in New York city who use drugs. J Infect Dis. 2008; 197(4):503-509. [PubMed: 18275272]

9. Cu-Uvin S, Ko H, Jamieson DJ, et al. Prevalence, incidence, and persistence or recurrence of trichomoniasis among human immuno-deficiency virus (HIV)-positive women and among HIVnegative women at high risk for HIV infection. Clin Infect Dis. 2002; 34(10):1406-1411. [PubMed: 11981738]

10. Gaydos CA, Hsieh YH, Barnes M, et al. Trichomonas vaginalis infection in women who submit self-obtained vaginal samples after Internet recruitment. Sex Transm Dis. 2011; 38(9):828-832. [PubMed: 21844738]

11. Nijhawan AE, DeLong AK, Celentano DD, et al. The association between Trichomonas infection and incarceration in HIV-seropositive and at-risk HIV-seronegative women. Sex Transm Dis. 2011; 38(12):1094-1100. [PubMed: 22082718]

12. Freeman AH, Katz KA, Pandori MW, et al. Prevalence and correlates of Trichomonas vaginalis among incarcerated persons assessed using a highly sensitive molecular assay. Sex Transm Dis. 2010; 37(3):165-168. [PubMed: 20023598]

13. Andrea SB, Chapin KC. Comparison of APTIMA Trichomonas vaginalis transcription-mediated amplification assay and BD affirm VPIII for detection of $T$. vaginalis in symptomatic women: Performance parameters and epidemiological implications. J Clin Microbiol. 2011; 49(3):866-869. [PubMed: 21248097]

14. Sutcliffe S, Newman SB, Hardick A, et al. Prevalence and correlates of Trichomonas vaginalis infection among female US federal prison inmates. Sex Transm Dis. 2010; 37(9):585-590. [PubMed: 20803782]

15. Sorvillo F, Smith L, Kerndt P, et al. Trichomonas vaginalis, HIV, and African-Americans. Emerg Infect Dis. 2001; 7(6):927-932. [PubMed: 11747718]

16. Centers for Disease Control and Prevention. [Accessed March 5, 2012.] STD rates by race or ethnicity. Available at: http://www.cdc.gov/std/health-disparities/race.htm

17. Adimora AA, Schoenbach VJ. Social context, sexual networks, and racial disparities in rates of sexually transmitted infections. J Infect Dis. 2005; 191(suppl 1):S115-S122. [PubMed: 15627221]

18. Sabol, W.; Minton, D.; Harrison, P. [Accessed March, 12, 2012.] Bureau of Justice Statistics Bulletin: Prison and jail inmates at midyear 2006; National Criminal Justice. 2007. p. 1-22.NCJ 217675Available at: http://www.ojp.usdoj.gov/bjs/abstract/pjim06.htm

19. Kissinger P, Amedee A, Clark RA, et al. Trichomonas vaginalis treatment reduces vaginal HIV-1 shedding. Sex Transm Dis. 2009; 36:11-16. [PubMed: 19008776]

20. Centers for Disease Control and Prevention. [Accessed March, 12, 2012] HIV surveillance in women. Available at: http://www.cdc.gov/hiv/topics/surveillance/resources/slides/women/ index.htm

21. Chesson HW, Blandford JM, Pinkerton SD. Estimates of the annual number and cost of new HIV infections among women attributable to trichomoniasis in the United States. Sex Transm Dis. 2004; 31(9):547-551. [PubMed: 15480116]

22. Munson E, Napierala M, Basile J, et al. Trichomonas vaginalis transcription-mediated amplification-based analyte-specific reagent and alternative target testing of primary clinical vaginal saline suspensions. Diagn Microbiol Infect Dis. 2010; 68(1):66-72. [PubMed: 20727473]

23. ACOG Committee on Practice Bulletins-Gynecology. ACOG Practice Bulletin no109: Cervical cytology screening. Obstet Gynecol. 2009; 114(6):1409-1420. [PubMed: 20134296]

24. Clarke JG, Phipps M, Rose J, et al. Follow-up of abnormal pap smears among incarcerated women. J Correctional Health Care. 2007; 13(1):22-26. 
25. Nijhawan AE, Salloway R, Nunn AS, et al. Preventive healthcare for underserved women: Results of a prison survey. J Womens Health (Larchmt). 2010; 19(1):17-22. [PubMed: 20088654]

26. Lara-Torre E, Pinkerton JS. Accuracy of detection of Trichomonas vaginalis organisms on a liquid-based Papanicolaou smear. Am J Obstet Gynecol. 2003; 188(2):354-356. [PubMed: 12592239]

27. Chapin K, Andrea S. APTIMA(R) Trichomonas vaginalis, a transcription-mediated amplification assay for detection of Trichomonas vaginalis in urogenital specimens. Expert Rev Mol Diagn. 2011; 11(7):679-688. [PubMed: 21902528]

28. Ghanem KG, Hutton HE, Zenilman JM, et al. Audio computer assisted self interview and face to face interview modes in assessing response bias among STD clinic patients. Sex Transm Infect. 2005; 81(5):421-425. [PubMed: 16199744]

29. Schachter J, Chernesky MA, Willis DE, et al. Vaginal swabs are the specimens of choice when screening for Chlamydia trachomatis and Neisseria gonorrhoeae: Results from a multicenter evaluation of the APTIMA assays for both infections. Sex Transm Dis. 2005; 32(12):725-728. [PubMed: 16314767]

30. Rathod SD, Krupp K, Klausner JD, et al. Bacterial vaginosis and risk for Trichomonas vaginalis infection: A longitudinal analysis. Sex Transm Dis. 2011; 38(9):882-886. [PubMed: 21844747] 


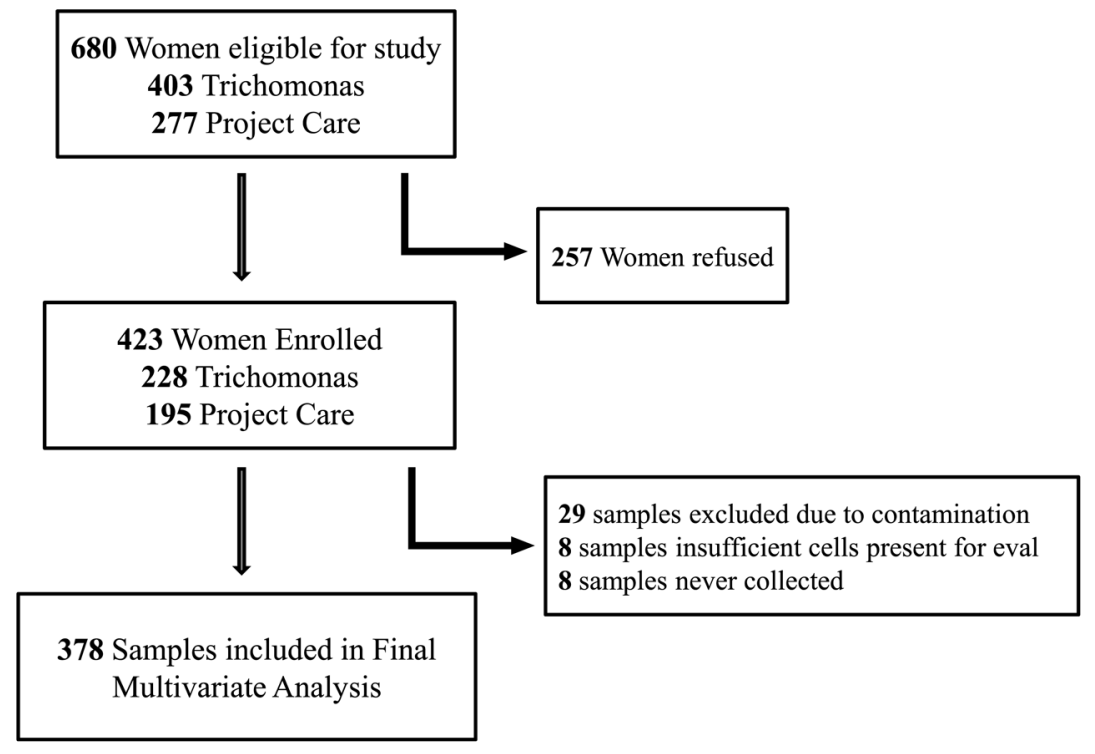

Figure 1.

Inclusion algorithm for all eligible subjects. 
TABLE 1

Baseline Characteristics by TMA Status

\begin{tabular}{|c|c|c|}
\hline & TMA Negative $(\mathbf{n}=325)$ & TMA Positive $(n=53)$ \\
\hline Age, mean (range), y & $31(22-49)$ & $29(19-55)^{*}$ \\
\hline \multicolumn{3}{|l|}{ Race/Ethnicity } \\
\hline White, non-Hispanic & $193(60 \%)$ & $28(57 \%)$ \\
\hline Black, non-Hispanic & $28(9 \%)$ & $10(20 \%)^{*}$ \\
\hline Hispanic & $56(17 \%)$ & $9(18 \%)$ \\
\hline Other (Native American, Pacific Islander, multiracial) & $45(14 \%)$ & $2(4 \%)$ \\
\hline Education $\geq 12 \mathrm{y} / \mathrm{GED}$ & $211(66 \%)$ & $27(55 \%)$ \\
\hline \multicolumn{3}{|l|}{ Marital status } \\
\hline Single, not living with partner & $132(41 \%)$ & $24(49 \%)$ \\
\hline Single, living with partner & $133(42 \%)$ & $19(39 \%)$ \\
\hline Married & $20(6 \%)$ & $0(0 \%)$ \\
\hline Separated & $14(4 \%)$ & $2(4 \%)$ \\
\hline Divorced & $18(6 \%)$ & $3(6 \%)$ \\
\hline Widowed & $3(1 \%)$ & $1(2 \%)$ \\
\hline Homeless & $30(9 \%)$ & $8(16 \%)$ \\
\hline \multicolumn{3}{|l|}{ Insurance } \\
\hline None & $140(44 \%)$ & $29(59 \%)$ \\
\hline Private & $63(20 \%)$ & $6(12 \%)$ \\
\hline Medicare/Medicaid & $116(36 \%)$ & $14(28 \%)$ \\
\hline \multicolumn{3}{|l|}{ Health care location } \\
\hline Private doctor & $112(35 \%)$ & $9(20 \%)$ \\
\hline Hospital clinic & $83(27 \%)$ & $10(21 \%)$ \\
\hline Community health center & $65(26 \%)$ & $12(21 \%)$ \\
\hline ED & $28(9 \%)$ & $9(20 \%)$ \\
\hline RIDOC & $4(1 \%)$ & $1(2 \%)$ \\
\hline Other & $25(8 \%)$ & $5(11 \%)$ \\
\hline \multicolumn{3}{|l|}{ Self-reported health status } \\
\hline Poor & $15(5 \%)$ & $4(8 \%)$ \\
\hline Fair & $76(24 \%)$ & $18(37 \%)$ \\
\hline Good & $153(48 \%)$ & $18(37 \%)$ \\
\hline Very good & $55(17 \%)$ & $4(8 \%)$ \\
\hline Excellent & $23(7 \%)$ & $5(10 \%)$ \\
\hline \multicolumn{3}{|l|}{ How long ago was your last Pap test? } \\
\hline Within 1 y & $212(67 \%)$ & $21(44 \%)$ \\
\hline $1-3$ y ago & $77(19 \%)$ & $19(40 \%)^{*}$ \\
\hline$>3 \mathrm{y}$ ago & $27(9 \%)$ & $8(17 \%)^{*}$ \\
\hline Ever been pregnant & $253(80 \%)$ & $42(85 \%)$ \\
\hline Ever miscarried & $104(32 \%)$ & $17(35 \%)$ \\
\hline
\end{tabular}




\begin{tabular}{|c|c|c|}
\hline & TMA Negative $(n=325)$ & TMA Positive $(n=53)$ \\
\hline Vaginal discharge & $75(38 \%)$ & $18(53 \%)$ \\
\hline Bleeding after intercourse & $17(9 \%)$ & $3(9 \%)$ \\
\hline Unusual/bad odor from vagina & $49(25 \%)$ & $9(26 \%)$ \\
\hline Pain with intercourse & $42(22 \%)$ & $10(29 \%)$ \\
\hline Vaginal itching & $23(12 \%)$ & $7(21 \%)$ \\
\hline Burning with urination & $22(11 \%)$ & $4(12 \%)$ \\
\hline Any symptoms & $133(41 \%)$ & $29(59 \%)^{*}$ \\
\hline \multicolumn{3}{|l|}{ Prior STIs/HIV } \\
\hline Chlamydia & $125(39 \%)$ & $24(49 \%)$ \\
\hline Gonorrhea & $53(17 \%)$ & $14(29 \%)$ \\
\hline Trichomonas & $65(20 \%)$ & $15(31 \%)$ \\
\hline Pelvic inflammatory disease & $40(14 \%)$ & $10(21 \%)$ \\
\hline Genital warts & $31(10 \%)$ & $5(10 \%)$ \\
\hline Herpes & $25(8 \%)$ & $4(8 \%)$ \\
\hline Syphilis & $4(1 \%)$ & $1(2 \%)$ \\
\hline HIV & $8(2 \%)$ & $1(2 \%)$ \\
\hline Any of the above & $186(58 \%)$ & $35(71 \%)$ \\
\hline \multicolumn{3}{|l|}{ Sexually active with } \\
\hline Men only & $221(69 \%)$ & $30(61 \%)$ \\
\hline Women only & $7(2 \%)$ & $1(2 \%)$ \\
\hline Men and women & $91(29 \%)$ & $18(37 \%)$ \\
\hline \multicolumn{3}{|l|}{ No. lifetime male sexual partners } \\
\hline $0-9$ & $187(58 \%)$ & $20(41 \%)$ \\
\hline$\geq 10$ & $135(42 \%)$ & $29(59 \%)^{*}$ \\
\hline Ever had sex for drugs or money & $71(22 \%)$ & $18(37 \%)^{*}$ \\
\hline Had main partner in 3 mo before incarceration & $274(85 \%)$ & $41(84 \%)$ \\
\hline \multicolumn{3}{|l|}{ Condom use with main partner } \\
\hline Every time & $13(4 \%)$ & $4(8 \%)$ \\
\hline Almost every time & $18(6 \%)$ & $5(10 \%)$ \\
\hline Almost half the time & $28(9 \%)$ & $3(6 \%)$ \\
\hline Almost never & $40(13 \%)$ & $4(8 \%)$ \\
\hline Never & $174(54 \%)$ & $25(51 \%)$ \\
\hline Had casual partner in 3 mo before incarceration & $110(34 \%)$ & $21(43 \%)$ \\
\hline \multicolumn{3}{|l|}{ Condom use with casual partner } \\
\hline Every time & $32(29 \%)$ & $7(33 \%)$ \\
\hline Almost every time & $24(22 \%)$ & $3(14 \%)$ \\
\hline Almost half the time & $21(19 \%)$ & $5(24 \%)$ \\
\hline Almost never & $12(11 \%)$ & $2(10 \%)$ \\
\hline Never & $20(18 \%)$ & $4(19 \%)$ \\
\hline \multicolumn{3}{|l|}{ Substance use in $30 \mathrm{~d}$ before incarceration } \\
\hline Alcohol to intoxication & $140(44 \%)$ & $21(43 \%)$ \\
\hline Heroin & $53(17 \%)$ & $7(14 \%)$ \\
\hline
\end{tabular}




\begin{tabular}{lcc}
\hline & TMA Negative $(\mathbf{n}=\mathbf{3 2 5})$ & TMA Positive $(\mathbf{n}=\mathbf{5 3})$ \\
\hline Other opiates & $80(25 \%)$ & $16(33 \%)$ \\
Cocaine & $104(33 \%)$ & $27(55 \%)^{*}$ \\
Benzodiazepines & $73(23 \%)$ & $12(24 \%)$ \\
Marijuana & $159(51 \%)$ & $26(55 \%)$ \\
Others (amphetamines, hallucinogens, inhalants, barbiturates) & $54(17 \%)$ & $8(16 \%)$ \\
Polysubstance use & $137(44 \%)$ & $27(56 \%)$ \\
Any substance use & $243(75 \%)$ & $41(84 \%)$ \\
\hline
\end{tabular}

NB. Percentages listed reflect the number of participants who answered the question.

Significant difference $(P<0.05)$ between TMA negative versus TMA positive (see Table 2 for details).

ED indicates emergency department. 


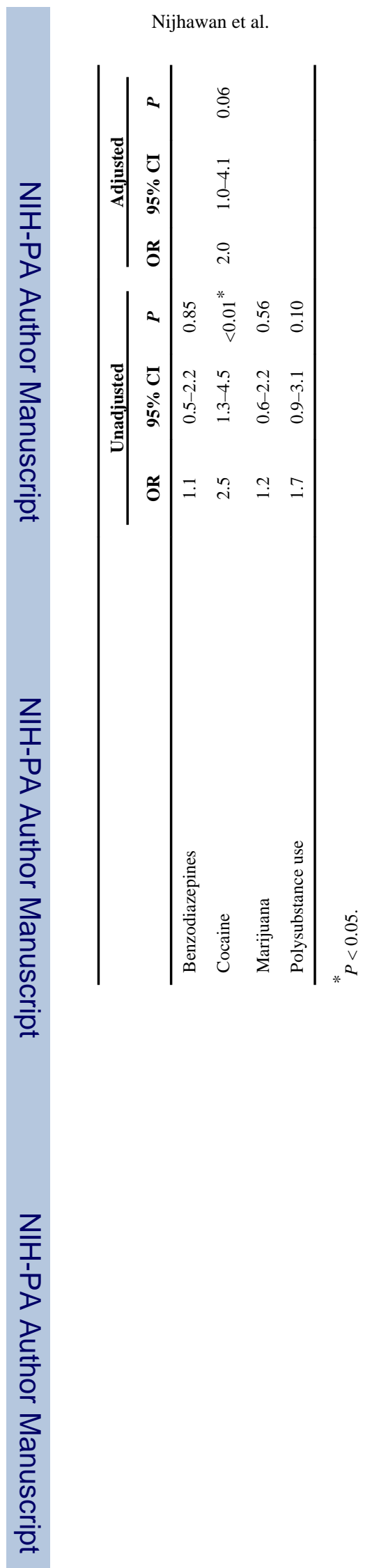

Sex Transm Dis. Author manuscript; available in PMC 2014 January 02. 